\title{
Wireless sensor network data fusion based on GSO improved BP algorithm
}

\author{
Fanjin Mai ${ }^{1, a^{*}}$, Huajian Huang ${ }^{1, b}$ \\ ${ }^{1}$ School of Mechanical \& Control Engineering, Guilin University of Technology, Guilin 541004, \\ China \\ agltide2010@126.com, bhhj-kk@163.com
}

Keywords: wireless sensor networks; data fusion; BP neural network; GSO algorithm.

Abstract: In order to improve the efficiency of data fusion in wireless sensor networks and reduce the energy consumption of the system, a new method of data fusion based on GSO algorithm for optimizing BP neural network is proposed. Sensor nodes sensing data, and then use the GSO algorithm to optimize the BP neural network data fusion. The simulation experiments results show that the proposed algorithm is better than the other typical algorithms and it improves the efficiency of data fusion, saves the energy consumption and prolongs the network life.

\section{Introduction}

Wireless sensor networks (WSN) are composed of a large number of wireless sensor nodes. Generally, sensor nodes are deployed in harsh environment, it is not easy to replace the battery. In order to improve the energy efficiency of the nodes, many scholars had carried out a lot of research on the data fusion routing algorithm based on WSNS. Some scholars try to apply BP neural network to the data fusion of wireless sensor networks and BP neural network. Lingyi Sun [1] proposed a data fusion algorithm based on neural network for wireless sensor network, which can effectively reduce the amount of network traffic and reduce the energy consumption of nodes. Glowworm Swarm Optimization algorithm (GSO)[2] is a new swarm intelligence algorithm, which is proposed by simulating the foraging behavior of the firefly. It not only has a strong global optimization ability, it is robust and easy to implement and it is suitable for optimizing the parameters of neural network.

On the basis of previous studies[3,4], this paper proposes an improved WSN data fusion algorithm,this algorithm optimizing neural networks with the firefly algorithm,we name it IGSO-BP.

\section{Glowworm Swarm Optimization algorithm and BP neural network algorithm}

\section{Glowworm Swarm Optimization}

Krishnanand and Ghose proposed the optimization algorithm of the Glowworm swarm in 2005[2]. In the basic glowworm swarm optimization algorithm, including the following 3 processes: fluorescence update, location update, the perception range update.

We suppose the number of the firefly is $\mathrm{N}$, the location of the ith firefly $\left(x_{i}, y_{i}\right)$ and the corresponding objective function is $f\left(x_{i}, y_{i}\right)$, and $\mathrm{T}_{\mathrm{i}}$ is the value of fluorescein, then the decision radius of each firefly is updated with the Eq.1:

$$
r_{d}^{i}(t+1)=\min \left\{r s, \max \left\{0, r_{d}^{i}(t)+\beta\left(n_{t}-\left|N_{i}(t)\right|\right)\right\}\right\}
$$

In the Eq. $1, \mathrm{R}_{\mathrm{d}}^{i}(t+1)$ is the decision radius of $n$th firefly in $(\mathrm{t}+1)$ generation; $\mathrm{r}_{\mathrm{s}}$ is the Sensing radius; $\beta$ is control parameter; $n_{t}$ is the number of firefly in the decision radius; $N_{i}(t)$ is the number of high fluorescence firefly in the decision radius, we use the following equation express $N_{i}(t)$ : 


$$
N_{i}(t)=\left\{j:\left\|x_{j}(t)-x_{i}(t)\right\|<R_{d}^{i} ; l_{i}(t)<l_{j}(t)\right\}
$$

In the Eq.2, $x_{j}(t)$ is the location of jth firefly in tth generation; $l_{j}(t)$ is the Value of fluorescein of jth firefly in th generation; the distance between the adjacent firefly is within the range of the visual field radius $\mathrm{R}_{\mathrm{d}}^{i}$. $p_{i j}(t)$ is the probability of the ith firefly move to the adjacent the jth firefly, we use the Eq.3 to explain it:

$$
p_{i j}(t)=\frac{l_{j}(t)-l_{i}(t)}{\sum_{k \in N_{i}(t)} l_{k}(t)-l_{i}(t)}
$$

The firefly position update equation is Eq. 4:

$$
x_{i}(t+1)=x_{i}(t)+s\left(\frac{x_{j}(t)-x_{i}(t)}{\square x_{j}(t)-x_{i}(t)}\right)
$$

In the Eq.4, $s$ is the step size. After the ith firefly moved to the new location, we recalculate the value of fluorescein by Eq. 5 .

$$
l_{i}(t+1)=(1-\rho) l_{i}(t)+\gamma f\left(x_{i}(t+1)\right)
$$

In this equation, $l_{\mathrm{i}}(t+1)$ is the ith firefly's value of fluorescein in $t+1$ iterations; $\rho \in(0,1)$ is a constant, the parameter that controls the value of the fluorescein, and it is related to the volatilization of fluorescein; $\gamma$ is the renewal rate of fluorescein; $f\left(x_{i}(t+1)\right)$ is the fitness function. In the neighbor set, when the ith firefly has found a higher value of fluorescein the jth firefly, and at this time if the distance between ith firefly and jth firefly is less than the decision radius, the ith firefly will move to the direction of the jth firefly in probability $p_{i j}(t)$; and then update the location by Eq. 4 and calculation objective function value of the new position. Finally according to Eq. 5 to update the fluorescence value.

\section{Application of BP neural network in WSN}

In the literature [1], the idea of BP neural network is applied to the data fusion of wireless sensor networks. We call it the BPDF algorithm(BPNN Data Fusion). This algorithm uses BP neural network to fuse the data from the nodes of the cluster head and the members. In each sensor node, the detected data is processed according to the input layer neural network function for the first step, and then the results are transmitted to other locations of cluster head. And then the cluster head processed the data according to the neuron function of the hidden layer and the output layer neuron function. Finally, the results are transmitted to the center of the sensor network. The model structure of the BPDF algorithm is shown in Fig.1.

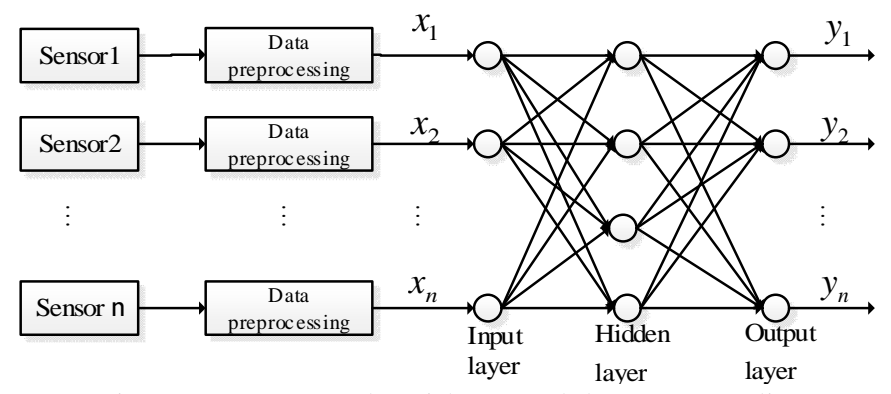

Figure 1 BPDF algorithm model structure diagram

\section{Application of GSO-BP algorithm in WSN}

The traditional BP algorithm has the disadvantages of slow convergence speed and easy to fall into local optimal solution. To solve this problem, optimizing the BP neural network with the GSO 
algorithm, which can improve the convergence speed and other performance of BP neural network. In this paper, the proposed algorithm is referred to as the IGSOBP algorithm. And the execution is divided into two steps:

(1) Network creation: Optimize the initial threshold and weight value of BP neural network by GSO algorithm, and then create a neural network; (2) Network training: Create a GSO-BP neural network, input the training data into the network, and then adjust the GSO-BP neural network's threshold and weight, when meeting the training requirements, stop iteration. The algorithm specific procedures are as follows:

1. Build the BP network topology and initialize the BP network threshold and weight.

2. Initialize GSO parameters and the training error of BP network is used as fitness value.

2.1 Update fluorescein

2.2 Update location

2.3 Calculating fitness value

2.4 Update decision

2.5 If meet the termination condition, output the value and use it as the weight and threshold of BP network. If not met, turn back to 2.1 and go on.

3. Calculate the training error, update the threshold and weight

4. Output the result if it meets the termination requirement and if not go back to step 2.

\section{Experimental settings and results}

\section{Simulation environment}

We had done programming experiments with MATLAB2014 on a computer with Pentium Dual Core CPU and 4GRAM. The GSO-BP algorithm parameter settings: the firefly number $m=100$, the intensity of the absorption coefficient $\gamma=1.0$, the maximum absorption $\beta=1.0$, the step size factor $\alpha=0.02$, the iteration number max $\mathrm{D}=200$. The simulation experiment node is provided as shown in table 1 .

Table 1. Experiment parameters

\begin{tabular}{c|l|l|l}
\hline Parameter & Value & Parameter & Value \\
\hline Network size & $100 * 100$ & $\begin{array}{l}\text { Receiving } \\
\text { consumption }\end{array}$ & $30 \mathrm{~nJ} / \mathrm{bit}$ \\
\hline Node number & 100 & Packet size & $100 \mathrm{bytes}$ \\
\hline Initial energy & $0.5 \mathrm{~J}$ & rounds & 1500 \\
\hline Communication range & $20 \mathrm{~m}$ & Simulation time & $500 \mathrm{~s}$ \\
\hline Transmission consumption & $50 \mathrm{~nJ} / \mathrm{bit}$ & & \\
\hline
\end{tabular}

\section{Experimental results and analysis}

In this paper, we had compared the performance of LEACH, BPNN, ABC-BP and IGSO-BP in WSN about their lifetime network energy consumption and data fusion efficiency.

Network lifetime

Comparison results of 4 algorithms for the survival of wireless sensor networks as shown in Fig.2. 


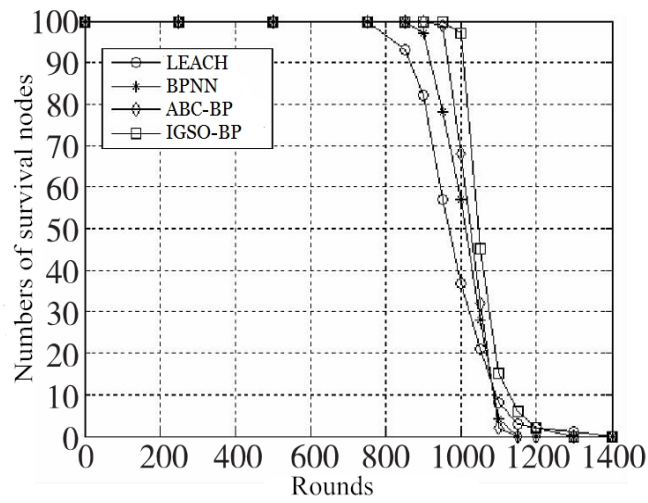

Figure2 Comparison of network node survival

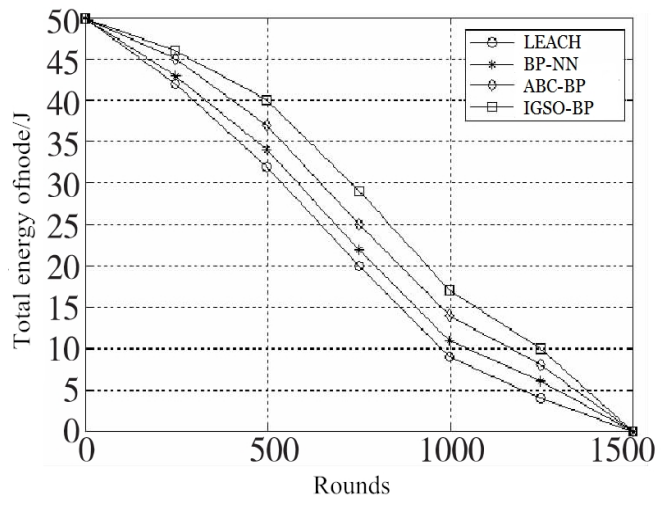

Figure 3 Network node residual energy

From Fig. 2, it can be seen that the number of nodes in each algorithm is beginning to appear to be different and f decreasing at round 800. From the difference we can see that the IGSO-BP algorithm has the longest life cycle and the best energy saving, its performance is better than other algorithms.

Overall energy consumption performance comparison The residual energy of nodes is one of the important evaluation indexes of Wireless Sensor Networks. As can be seen from Fig.3, with the increase of network running time, the remaining energy of the nodes of the 4 algorithms are reducing, and the energy consumption of leach is the largest, and the energy consumption of IGSO-BP is best, the performance of IGSO-BP is better than ABC-BP and BPNN.

Performance of data fusion rate

From Fig.4 it can be seen that the efficiency of network data fusion is gradually improving with the increase of the number of nodes in the network. Test results show that proposed IGSO algorithm has a better performance of data fusion in network than ABC-BP algorithm and BPNN algorithm. So the proposed IGSO-BP algorithm can improve the efficiency of data fusion in Wireless Sensor Network.

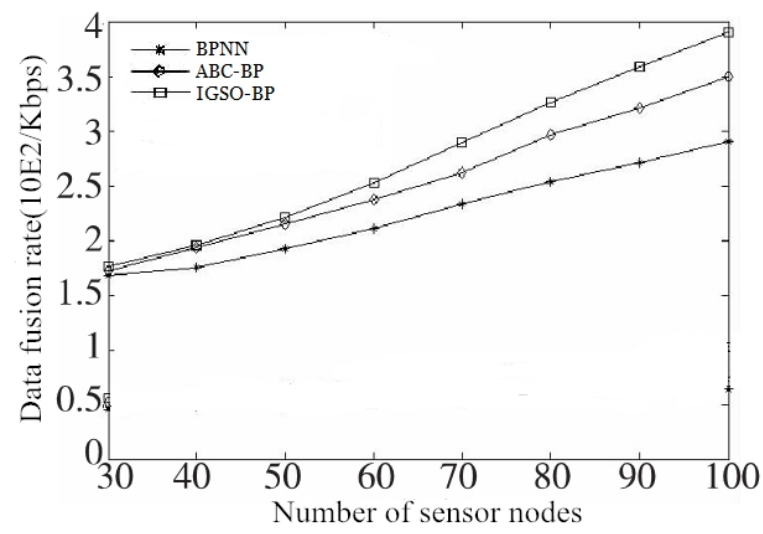

Figure 4 Comparison of data fusion efficiency

\section{Conclusions}

According to the specific environment needs of WSN, this paper had proposed an improved algorithm (IGSO-BP) to promote the efficiency of data fusion in Wireless Sensor Network. Experimental results show that proposed algorithm is highly competitive with standard BPNN algorithm and other algorithm. Reduce the energy consumption of nodes and prolong the network life cycle, and improving the performance of data transmission in Wireless Sensor Networks. 


\section{References}

[1] Lingyi Sun, Xianxiang Huang, Cai Wei. Data fusion algorithm based on wireless sensor network based on neural network [J]. Journal of sensing technology .2011,24 (1);123 - 127.

[2] Krishhand K N, Ghose D. Glowworm swarm optimization for simultaneous capture of multiple local optimal of multimodal functions [J].SwarmIntelligence, 2009, 3(2): 87 -124.

[3] Yue Hou, He Zhao. BP neural network algorithm based on the optimization of firefly [J]. Journal of Lanzhou University.2013,32(6);25-28

[4] Qiuhong Chen, Meng Guo. The algorithm of wireless sensor network data fusion based on PSO-BP[J]. Computer measurement and control. 2014.22(4) 